\title{
DIVISION VIII: GALAXIES AND THE UNIVERSE
} (LES GALAXIES ET L'UNIVERS)

\author{
PRESIDENT: Virginia Trimble \\ BOARD: F. Bertola, M. Birkinshaw, S. Lilly, S. Okamura, J. Peacock, E. \\ Sadler, P. Shaver, A. Szalay
}

Commission 28: Galaxies

Commission 47: Cosmology

\section{Report of Business Meeting, July 21, 2003}

Attendance: F. Bertola, E. M. Corsini, A. Fairall, W. Hillebrandt, J. Huchra, Y. Jing, J. Jugaku, E. Khachikian, P. Mazzali, K. Nomoto, D. O'Donoghue, S. Okamura, B. Partridge, E. Sadler, M. Salvo, B. Schmidt, P. Shaver, S. Simkin, V. Trimble, B. Tully, M. Turatto, R. Webster, H. Yamaoka

This was a joint meeting of Division VIII and its two Commissions, 28 and 47. The Commission reports, including approval of new officers and committee members, appear separately, immediately following this.

Division VIII Officers. Triennium 2003-2006: The Division Board includes the Presidents, vice Presidents, and past Presidents of the two Commissions, a Division President and Vice President (who normally becomes President) and Webmaster (Secretary under the new bylaws), some of whom may also be past Commission Presidents, plus the past president of the Division.

President: $\quad$ F. Bertola

Vice President: S. Okamura (past President, Commission 28)

Webmaster: $\quad$ M. Birkinshaw

Board: $\quad$ E. Sadler (President, Commission 28)

F. Combes (Vice President, Commission 28)

J. Peacock (Past President, Commission 47)

S. Lilly (President, Commission 47)

R. Webster (Vice President, Commission 47)

V. Trimble (Past President, Division VIII)

\section{Working Groups}

A proposal for the establishment of a Working Group on Computational Astrophysics was received too late for formal action, but participants expressed general support for the idea. The new president and board will submit the formal proposal to the Executive Committee later for approval.

An inaugural meeting of the re-established Working Group on Supernovae, which had been formally approved by the EC during the triennium, took place. There were 14 participants. The re-establishment was suggested at a supernova meeting in Garching, 
Germany in August 2002, with preliminary goals of coordinating observational activities, establishing an electronic newsletter, and assisting with the archiving of data and its accessibility. Wolfgang Hillebrandt (Germany) was elected chair and Brian Schmidt (Australia) vice-chair for the period 2003-2006. Paolo Mazzali acted as secretary.

The creation of an e-newsletter was found to the first and perhaps most urgent task and it is hoped that a start can be made at Trento SNIa meeting in September, 2003. It could be useful for both scientific and outreach purposes. A format needs to be decided. The gamma ray burst network (GCN) system is a possible model for announcing supernovae discoveries and early data.

Announcement and designation of names for supernovae. At present this is done via IAU Circulars, which involves some delay with the benefit of reasonable reliability, since naming required spectroscopic confirmation or all three of known position, a second (independent) observation, and a "history" (initial light curve), according to D. Green who heads the IAU Telegram office. Rapid announcement increases the opportunities for quick follow-up, but also for errors. One possibility is initial announcement via the Working Group e-newsletter, followed, if appropriate, by a formal IAU Circular announcement and designation. A central server will be needed and, perhaps, some system of preliminary designation (as is currently done for comets and asteroids before their orbits have been determined). The present system of formal designations will need to be reconsidered before the number of annual discoveries reaches 700 . B. Schmidt volunteered to look into these issues.

Follow-up observations. Thought needs to be given to how the increasing number of groups can coordinate these. A robotic telescope (for which funding might conceivably be found in Germany) is one possibility. The data would probably be proprietary.

Data archiving and availability. The Hubble Space Telescope Treasury Program is a possible model., but spectra, non-optical data, and polarimetry should also be included. There are already supernova data repositories connected with some searches (Asiago and Lick, for instance). These might be expanded to include data from other sources and made accessible through the International Virtual Observatory. Reference models of explosions, light curves, and spectra could also be a valuable part of such archives.

\section{Scientific Meetings}

Symposia 216, 217, and 220 (whose proceedings appear in separate volumes) and Joint Discussions $6,10,13,15,18$, and 21, and Special Session 2 (whose proceedings appear in Highlights) pertained to the topics of galaxies and the universe. Most of these were formally sponsored or co-sponsored by the Division and/or its Commissions.

In addition, the Division sponsored a meeting on the GRB-Supernova Connection on 18 July 2003. details of the program were, deliberately, established very late. This prevented them from appearing in the formal GA program but did allow the meeting to include work on the March 2003 event GRB 030329 and its corresponding supernova. The Division plans similar sessions on late-breaking topics for future General Assemblies. The presentations (coordinated by E. Sadler and B. Schmidt) concerned observations and models of supernovae, hypernovae (supernovae with very broad absorption features, which suggest high-velocity ejecta, and unusually energetic explosions), and gamma-ray bursts.

M. Iye (NAOJ) reported on recent Subaru observations of SN 2002ap (a type Ic core collapse supernovae in the nearby galaxy NGC 628) and GRB 030329 (identified with SN 2003dh in a galaxy at $z=0.16$ ). Both were optically polarized, and in SN 2002ap the presence of a redshifted polarized component hints at the presence of a relativistic jet. 
K. Nomoto (Tokyo) gave a general overview of the properties of hypernovae from a theoretical perspective, noting that the explosion kinetic energy can exceed $5-10 \times 10^{51}$ ergs. Core-collapse supernovae can form black holes from stars with initial masses of $20-25$ solar masses or more. Metallicity effects are almost certainly important: lowmetallicity environments, such as those in the early universe and in the star-forming dwarf galaxies that are seen as GRB hosts favor GRB production.

S. van Dyk (IPAC) reviewed recent work on the radio emission from supernovae, concluding that most type Ib and Ic events are not associated with GRBs, while S. Ryder (JAC, Hawaii) discussed the radio monitoring of the type IIb SN 2001ig, where there is evidence for a variable mass-loss rate from the progenitor stars, possibly because it was in a binary system.

G. Chincarini (Milano) described the SWIFT mission, due for launch in 2004, which should detect at least $100 \mathrm{GRBs}$ per year. A ground-based IR telescope at La Silla (REM) will be used for follow-up imaging and there are opportunities for other observatories to take part.

There were two papers on SN 1987A, from L. Staveley-Smith (ATNF) on the radio evolution of the remnant (including new high-resolution images from the Australia Telescope Compact Array at $18 \mathrm{GHz}$ ), and from R. Kirshner (Harvard) on the optical evolution (including new images from ACS on HST). B. Schmidt (ANU) reported the latest result results on the high-redshift Type Ia supernovae, noting that more than 200 supernovae have now been found in the redshift range $z=0.01-1.2$. The final talk was by N. Yamaoka on GRB afterglows, noting that optical light curves of these can show unexpected behavior, such as plateaus, on time-scales of hours, so that continuous photometry over days is needed to improve our understanding of them.

Virginia Trimble

President of the Division 